\title{
Transcriptional regulation of drug-resistance genes in Candida albicans biofilms in response to antifungals
}

\author{
T. Watamoto, ${ }^{1,2}$ L. P. Samaranayake, ${ }^{1}$ H. Egusa, ${ }^{2}$ H. Yatani ${ }^{2}$ \\ and C. J. Seneviratne ${ }^{1}$ \\ ${ }^{1}$ Oral Biosciences, Faculty of Dentistry, The University of Hong Kong, Hong Kong SAR \\ ${ }^{2}$ Department of Fixed Prosthodontics, Osaka University Graduate School of Dentistry, Osaka, Japan
}

Correspondence

C. J. Seneviratne

jaya@hku.hk

Received 25 January 2011

Accepted 2 April 2011

\section{INTRODUCTION}

Candida albicans is the major fungal pathogen causing infections in humans, ranging from superficial mucosal infection to systemic mycoses (Samaranayake \& MacFarlane, 1990). Candidal infections rank as the third or fourth leading cause of hospital-acquired infection in the USA and are associated with high morbidity and mortality rates (Pfaller \& Diekema, 2007). The ability of free-floating (planktonic) cells to exhibit an attached biofilm mode of growth is a major virulence attribute of Candida species and a direct cause of therapeutic failure (Tumbarello et al., 2007). As with other microbial biofilms, a key phenotypic feature of Candida biofilms is their high degree of drug resistance (Seneviratne et al., 2008a). Therefore, a thorough understanding of Candida biofilm properties is of great clinical importance.

In general, four classes of antifungal drugs are used to manage fungal infections, namely polyenes, azoles, fluoropyrimidines

Abbreviation: qRT-PCR, quantitative real-time RT-PCR. and echinocandins (Ruhnke et al., 2008). One mechanism by which C. albicans acquires increased antifungal resistance is the expression of drug-resistance genes. For instance, the expression of genes regulating drug efflux pumps, which expel a wide range of toxic metabolites and drugs out of cells, is associated with increased drug resistance against azole antifungals in the planktonic mode of C. albicans (Prasad \& Kapoor, 2004). Acquired drug resistance in Candida is related to ATP-binding cassette transporters encoded by the Candida drug-resistance genes $C D R 1$ and $C D R 2$, as well as major facilitator transporters encoded by the multidrug-resistance gene MDR1 (Nakamura et al., 2001). Resistance of C. albicans against azole antifungals is associated with overexpression or mutation of ERG11, which encodes 14- $\alpha$-demethylase in the ergosterol biosynthesis pathway. In contrast, echinocandins are poor substrates for multidrug efflux transporters, and resistance is commonly associated with point mutations or overexpression of the FKS1 gene (Nett et al., 2010).

Most of the published studies examining the transcriptional expression of drug-resistance genes in C. albicans have been 
confined to the planktonic mode of growth, and few data are available for the biofilm mode (Franz et al., 1998, 1999; Holmes et al., 2008; Lopez-Ribot et al., 1998; Perea et al., 2001; Sanglard et al., 1995; White, 1997). Therefore, we aimed to study the influence of mode of growth (planktonic, adhesion and biofilm) on the transcriptional expression of several genes that are associated with drug resistance in $C$. albicans. In addition, we included the PIL1 gene, which encodes the hyphal-specific echinocandin-binding protein (Pillp), as we found previously that Pillp is significantly upregulated in Candida biofilms and have proposed it as a possible drug target against Candida biofilms (Seneviratne et al., 2008c). In the present study, we also tested the response of C. albicans biofilms to three classes of antifungals, namely azoles, polyenes and echinocandins.

\section{METHODS}

Organisms and culture conditions. C. albicans strain SC5314, which is well characterized and has been used in our previous studies (Jayatilake et al., 2007; Lu et al., 2006), was subcultured on Sabouraud dextrose agar (SDA; Gibco) and maintained at $4{ }^{\circ} \mathrm{C}$ during the experimental period. The purity of the cultures was confirmed periodically by Gram staining and the use of commercially available carbohydrate assimilation tests (API 32C identification system; bioMérieux).

Preparation of Candida cultures in recently adhered, biofilm and planktonic modes. Candida cultures in the different modes of growth were prepared according to a previously published protocol (Jin et al., 2004). In brief, Candida cells were grown in SDA medium at $37{ }^{\circ} \mathrm{C}$ for $18 \mathrm{~h}$. A loopful of yeast was inoculated into a flask of yeast nitrogen base (YNB; Difco) medium supplemented with $50 \mathrm{mM}$ glucose and incubated in a rotary shaker at 75 r.p.m. overnight. Cells were washed twice with $20 \mathrm{ml}$ PBS $(0.1 \mathrm{M}$, pH 7.2) and resuspended in YNB supplemented with $100 \mathrm{mM}$ glucose until it reached an $\mathrm{OD}_{520}$ of $0.38\left(1 \times 10^{7} \mathrm{cells}^{-1}\right)$. This standard cell suspension was used to obtain recently adhered, biofilm and stationary-phase planktonic cultures. First, $100 \mu \mathrm{l}$ suspension was placed into each well of a 96-well microtitre plate and incubated for $90 \mathrm{~min}$ at $37^{\circ} \mathrm{C}$ in a shaker at 75 r.p.m. to allow yeast adherence to the well surface. The growth medium was aspirated and each well was washed with PBS to remove loosely adherent cells, leaving cells in the 'recently adhered mode' of growth, to which YNB containing $100 \mathrm{mM}$ glucose was added. Plates were then incubated at $37{ }^{\circ} \mathrm{C}$ in a shaker at 75 r.p.m. for $24 \mathrm{~h}$ to obtain cultures in biofilm mode. A portion of the standard cell suspension was incubated under identical conditions for $24 \mathrm{~h}$ to obtain stationary-phase planktonic cultures. Stationary-phase planktonic cultures reached a concentration of approximately $10^{8}$ cells $\mathrm{ml}^{-1}$ by $24 \mathrm{~h}$ and were considered to be high-density planktonic cultures. For each growth phase, microscopic examination of the cultures was performed to rule out contamination.

Antifungal susceptibility testing of stationary-phase planktonic, recently adhered and biofilm cultures. The commonly used antifungal agents amphotericin B, caspofungin and ketoconazole, belonging to the polyene, echinocandin and azole classes of antifungals, respectively, were used in this study. The antifungal agents were prepared as described previously (Ellepola \& Samaranayake, 2001). In brief, stock solutions of each drug were serially diluted with RPMI 1640 (Life Technologies) supplemented with $2 \%$ glucose to obtain drug concentrations ranging from 480 to $0.225 \mu \mathrm{g} \mathrm{ml}^{-1}$ for amphotericin $\mathrm{B}$, 100 to $0.1 \mu \mathrm{g} \mathrm{ml}^{-1}$ for caspofungin and 64 to $0.125 \mu \mathrm{g} \mathrm{ml}{ }^{-1}$ for ketoconazole. Antifungal susceptibility testing was performed using an XTT reduction assay, as described previously (Seneviratne et al., 2008b). In brief, $24 \mathrm{~h}$ Candida biofilms were washed with $100 \mu \mathrm{l}$ PBS to remove non-adherent cells. Each drug solution $(100 \mu \mathrm{l})$ was added to the microtitre plate containing Candida biofilms. Biofilms were then incubated at $37^{\circ} \mathrm{C}$ for $24 \mathrm{~h}$ with the antifungals and the metabolic activity of the fungal cells was determined by XTT assay. XTT (Sigma) solution ( $1 \mathrm{mg} \mathrm{ml}^{-1}$ in PBS) was prepared, filter-sterilized through a $0.22 \mu \mathrm{m}$ pore size filter and stored at $-70{ }^{\circ} \mathrm{C}$. Menadione (Sigma) solution $(0.4 \mathrm{mM})$ was prepared and filter-sterilized immediately before each assay. Prior to each assay, XTT solution was thawed and mixed with the menadione solution at a ratio of $5: 1$ by volume. The biofilms were first washed with $200 \mu \mathrm{l}$ PBS, and then $200 \mu \mathrm{l}$ XTT solution (PBS: XTT: menadione at $158: 40: 2$ ) was added to each of the pre-washed wells and the control wells. High-density planktonic cultures exposed to antifungals in microtitre plates were mixed directly with $200 \mu \mathrm{XTT}$ solution, as described in previous studies (Seneviratne et al., 2008b). Microtitre plates with biofilm and high-density planktonic cultures were then incubated in the dark for $3 \mathrm{~h}$ at $37^{\circ} \mathrm{C}$. Following incubation, $100 \mu \mathrm{l}$ solution was transferred to new wells and the colour change in the solution was measured with a microtitre plate reader (SpectraMAX 340 Tunable Microplate Reader; Molecular Devices) at $490 \mathrm{~nm}$. The MIC value was defined as the lowest drug concentration with a $50 \%$ reduction in opacity $\left(\mathrm{MIC}_{50}\right)$ compared with the drug-free control. Each experiment was repeated three times.

Transcriptional regulation of drug-resistance genes. To study the transcriptional regulation of genes associated with drug resistance in C. albicans (CDR1, CDR2, MDR1, ERG11, FKS1 and PIL1), we cultured cells in recently adhered and biofilm modes as described above but in polystyrene six-well plates instead of in 96-well plates (Seneviratne et al., 2008c). Adhered Candida cells were collected from six-well plates after $1.5 \mathrm{~h}$ (recently adhered mode) and $24 \mathrm{~h}$ (biofilm mode), washed with PBS and processed for RNA extraction. In parallel, $24 \mathrm{~h}$ stationary-phase planktonic cultures of C. albicans were harvested and total RNA was obtained. We also examined transcriptional regulation in $24 \mathrm{~h} \mathrm{C}$. albicans biofilms in response to antifungal treatment. Candida biofilms were incubated for $24 \mathrm{~h}$ with subMIC concentrations of antifungals $\left(1.88 \mu \mathrm{g}\right.$ amphotericin $\mathrm{B} \mathrm{ml}{ }^{-1}$, $20 \mu \mathrm{g}$ caspofungin $\mathrm{ml}^{-1}$ and $32 \mu \mathrm{g}$ ketoconazole $\mathrm{ml}^{-1}$ ). Cells were then washed with PBS and processed for RNA extraction.

RNA extraction, cDNA synthesis and quantitative real-time PCR. Total RNA was extracted from Candida cultures using the SV Total RNA isolation system (Promega) according to the manufacturer's instructions (Samaranayake et al., 2005). RNA purity and integrity were determined with a NanoDrop ND-1000 spectrophotometer (NanoDrop Technologies). Additionally, gel electrophoresis was performed to verify that the RNA was intact. cDNA was synthesized from $2 \mu \mathrm{g}$ total RNA using oligo(dT) primers and Superscript III reverse transcriptase (Invitrogen), as described previously (Seneviratne et al., 2008c). For quantitative analysis of gene expression, the mRNA level was measured using quantitative real-time RT-PCR (qRT-PCR). The primers (Sigma-Aldrich) used in this assay are listed in Table 1. qRT-PCR was carried out using an ABI PRISM 7900HT (Applied Biosystems) sequence detection system using SYBR Green incorporation (SYBR Green PCR Master Mix; Applied Biosystems) in duplicate for at least three separate experiments. Twenty microlitres of PCR mix $(10 \mu \mathrm{l}$ SYBR PCR Master Mix, $1 \mu \mathrm{l} \mathrm{cDNA}, 2 \mu \mathrm{l}$ primer mix and $7 \mu \mathrm{l}$ double-distilled water) was used for each gene and qRT-PCR was performed using the following cycling conditions: $95{ }^{\circ} \mathrm{C}$ for $5 \mathrm{~min}$, followed by 40 cycles of $95{ }^{\circ} \mathrm{C}$ for $15 \mathrm{~s}$ and $60{ }^{\circ} \mathrm{C}$ for $30 \mathrm{~s}$. Fluorescence intensities were quantified using StepOne software (Applied Biosystems). The relative quantities of the target genes were normalized against ACT1 housekeeping gene expression and analysed using the comparative $\Delta \Delta C_{\mathrm{t}}$ method, taking the amplification efficiency into 
Table 1. Gene-specific primers used for qRT-PCR

F, Forward; R, reverse.

\begin{tabular}{|c|c|c|}
\hline $\begin{array}{l}\text { Target } \\
\text { gene }\end{array}$ & Sequence $\left(5^{\prime} \rightarrow 3^{\prime}\right)$ & $\begin{array}{l}\text { Amplicon } \\
\text { size (bp) }\end{array}$ \\
\hline$A C T 1$ & $\begin{array}{l}\text { F: GCTTTTGGTGTTTGACGAGTTTCT } \\
\text { R: GTGAGCCGGGAAATCTGTATAGTC }\end{array}$ & 72 \\
\hline$C D R 1$ & $\begin{array}{l}\text { F: GTACTATCCATCAACCATCAGCACTT } \\
\text { R: GCCGTTCTTCCACCTTTTTGTA }\end{array}$ & 79 \\
\hline$C D R 2$ & $\begin{array}{l}\text { F: TGCTGAACCGACAGACTCAGTT } \\
\text { R: AAGAGATTGCCAATTGTCCCATA }\end{array}$ & 104 \\
\hline$M D R 1$ & $\begin{array}{l}\text { F: TCAGTCCGATGTCAGAAAATGC } \\
\text { R: GCAGTGGGAATTTGTAGTATGACAA }\end{array}$ & 91 \\
\hline$F K S 1$ & $\begin{array}{l}\text { F: CGTGAAATTGATCATGCCTGTAC } \\
\text { R: AACCCTTCTGGGCTCCAAA }\end{array}$ & 71 \\
\hline ERG11 & $\begin{array}{l}\text { F: GGTGGTGATTTGAATGATTTGACTTAT } \\
\text { R: GGCATATGCATTCTAAGAGTTTCCT }\end{array}$ & 92 \\
\hline PIL1 & $\begin{array}{l}\text { F: TAAGCAATTGAGTGCTTGGG } \\
\text { R: GGTTGGACAGAACCTTCGAT }\end{array}$ & 174 \\
\hline
\end{tabular}

consideration (Pfaffl, 2001). Groups were compared by analysis of variance with the significance level set at $P<0.01$.

\section{RESULTS}

\section{Antifungal susceptibility testing}

Biofilm C. albicans cultures were generally more resistant to antifungals than recently adhered cells and stationary-phase, high-density planktonic cultures (Table 2), as reported in previous studies (Baillie \& Douglas, 1999; Chandra et al., 2001; Seneviratne et al., 2008b). However, all cultures, including stationary-phase, high-density planktonic cultures, were resistant to ketoconazole. This is in agreement with previous studies on the dose-dependent response of fungistatic drugs (Seneviratne et al., 2008b; Beggs, 1989).

\section{Transcriptional expression of drug resistance- associated genes in different growth modes}

Transcriptional expression of CDR1, CDR2, MDR1, ERG11 and FKS1 was lower in recently adhered C. albicans cultures than in stationary-phase planktonic cultures (Fig. 1a-e). In contrast, expression of PIL1 was significantly increased in recently adhered cells (Fig. 1f; $P<0.01$ ). There was no significant difference between stationary-phase cultures and biofilm cultures in terms of expression of CDR1, CDR2, MDR1, FKS1 and ERG11. Interestingly, expression of PIL1 was significantly higher in the biofilm cultures than in the planktonic cultures $(P<0.01)$.

\section{Transcriptional regulation of drug resistance- associated genes in Candida biofilms in response to antifungals}

Expression of CDR1 was not significantly altered when biofilms were challenged with antifungal agents (Fig. 2a). $C D R 2$ expression increased significantly on caspofungin challenge (Fig. 2b). Interestingly, MDR1 expression was greatly increased on amphotericin B challenge but not with other drugs (Fig. 2c). Caspofungin and amphotericin B significantly increased the expression of FKS1 (Fig. 2d; $P<0.01$ ). Caspofungin upregulated FKS1 expression to a greater extent than ketoconazole and amphotericin $\mathrm{B}$, but the difference was not statistically significant $(P>0.05)$. Of all the antifungals tested, only ketoconazole significantly upregulated ERG11 expression (Fig. 2e; $P<0.01$ ). In contrast, amphotericin $B$ and caspofungin significantly downregulated PIL1 expression (Fig. 2f; $P<0.01$ ).

\section{DISCUSSION}

C. albicans is the major fungal pathogen of humans (Navarro-García et al., 2001). In recent years, Candida infections have increased disproportionately as a result of the increased number of compromised host populations, such as patients with AIDS, diabetes and various cancers, and organ-transplant recipients (Beck-Sagué et al., 1993; Wisplinghoff et al., 2004). The number of drug-resistant Candida strains has also increased dramatically owing to the increased use of antifungal agents. The expression of drug-resistance genes as a mechanism by which Candida acquires drug resistance is a well-known phenomenon in the planktonic mode of growth of this fungus. In the present study, we examined the transcriptional regulation of a subset of drug-resistance genes associated with drug resistance in C. albicans under various growth modes and when biofilms were exposed to antifungals.

We found that adhered cells of C. albicans were more resistant to antifungals than stationary-phase, high-density planktonic cultures, and resistance increased further after the formation of biofilms. This phenomenon has been

Table 2. Antifungal susceptibility testing of C. albicans planktonic, recently adhered and biofilm growth modes

\begin{tabular}{|lccc|}
\hline Antifungal agent & \multicolumn{2}{c|}{${\text { MIC }\left(\boldsymbol{\mu g ~ m l} \mathbf{~}^{-\mathbf{1}}\right)}$} & \\
\cline { 2 - 3 } & Stationary-phase, planktonic culture & Recently adhered mode & Biofilm mode \\
\hline Amphotericin B & 1.8 & 7.5 & 30 \\
Caspofungin & 0.25 & 0.5 & $>100$ \\
Ketoconazole & $>128$ & $>128$ & $>128$ \\
\hline
\end{tabular}


(a)

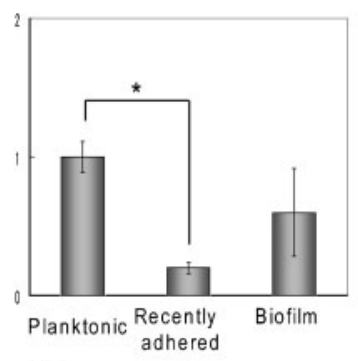

(d) FKS 1

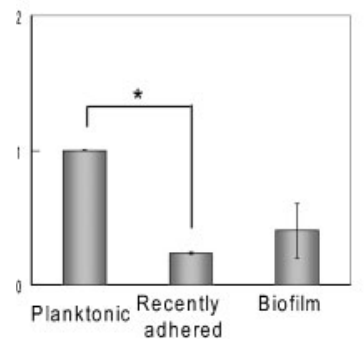

(b)

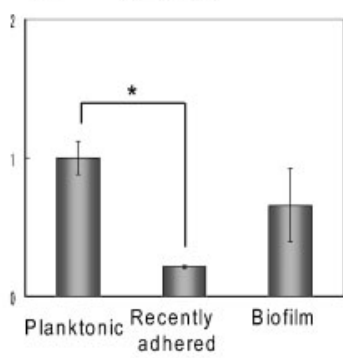

(e) ERG11

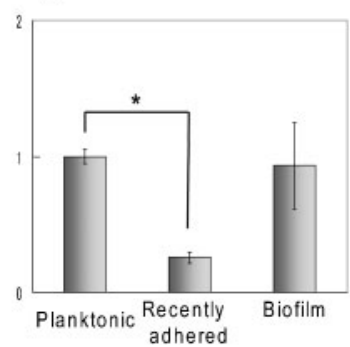

(c)

$M D R 1$

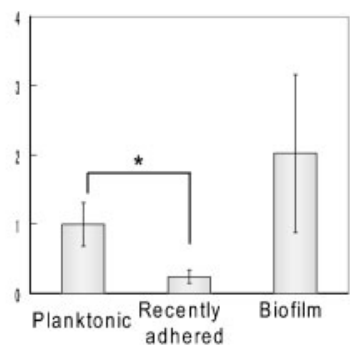

(f) $\quad$ PIL 1

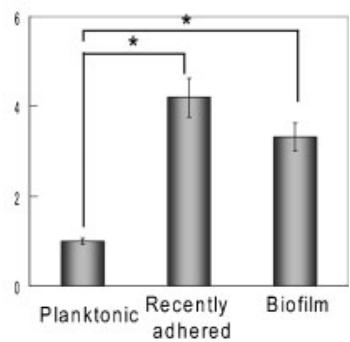

Fig. 1. Relative transcriptional expression of drug-resistance genes in different growth modes of $C$. albicans. Results were normalized against ACT1 housekeeping gene expression, which was assigned a value of 1 , and are shown as means $\pm S D$ of three independent experiments. ${ }^{*}, P<0.01$.

observed by various research groups, including ourselves (Mateus et al., 2004; Seneviratne et al., 2008b). However, levels of expression of the drug efflux pump-related genes
$C D R 1, C D R 2$ and MDR1 were significantly lower in adhered cells than in planktonic cultures. Therefore, it is unlikely that efflux pump-related genes play a major role in (a) $\quad C D R 1$

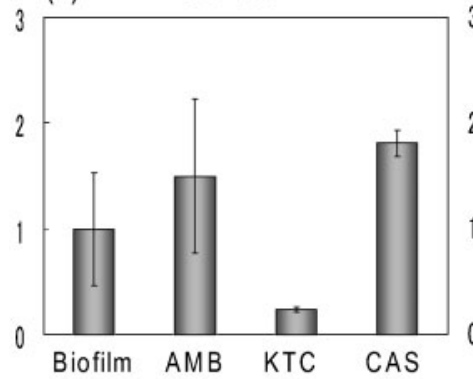

(d) FKS 1

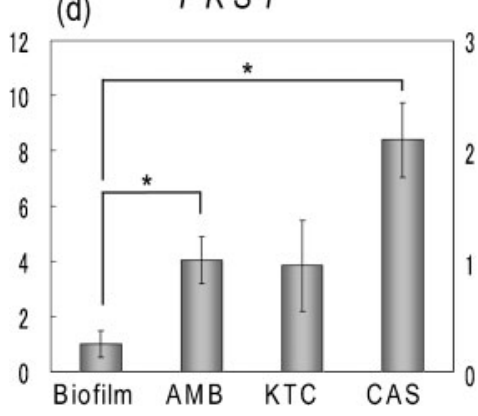

(b) $\quad C D R 2$

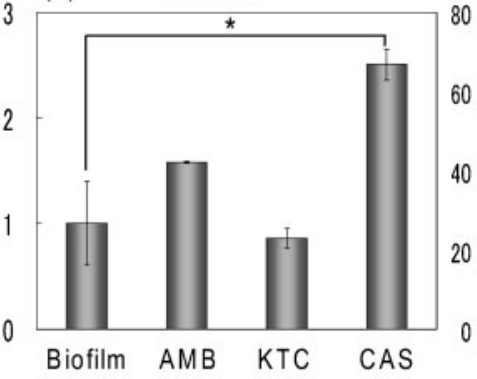

(e) ERG11

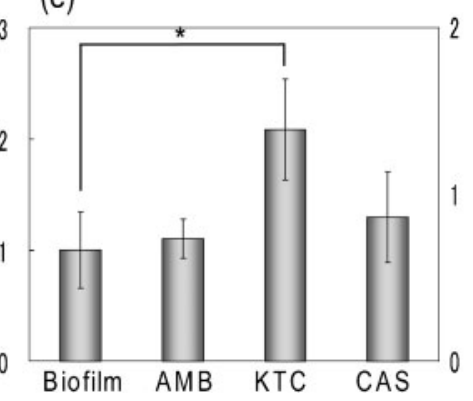

(c) MDR1

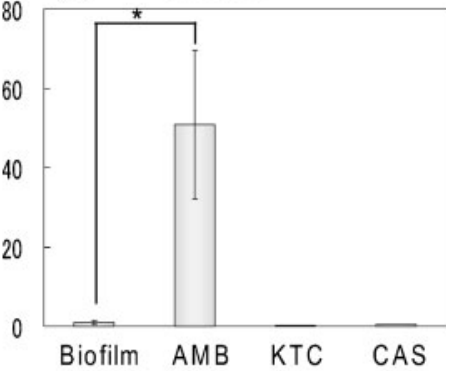

(f) $\quad$ PIL 1

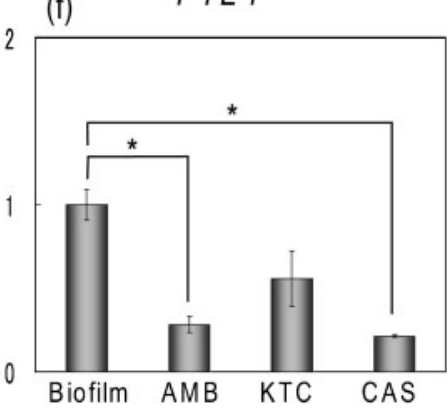

Fig. 2. Relative transcriptional expression of drug-resistance genes in C. albicans biofilms in response to antifungal agents. Results were normalized against $A C T 1$ housekeeping gene expression, which was assigned a value of 1 , and are shown as means $\pm \mathrm{SD}$ of three independent experiments. AMB, Amphotericin B; KTC, ketoconazole; CAS, caspofungin. ${ }^{*}, P<0.01$. 
adhesion-conferred drug resistance of C. albicans. Our results are in agreement with those of other studies, which found that multidrug efflux pumps have a relatively minor role in the mechanism of resistance of $C$. albicans biofilms against fluconazole, an azole drug (Mukherjee et al., 2003; Ramage et al., 2002). However, our study demonstrates the minor involvement of efflux pumps in resistance to polyene and echinocandin classes of drugs.

Mukherjee et al. (2003) observed that sterol composition, particularly ergosterol, is significantly decreased in the late phase of C. albicans biofilm formation. Thus, they proposed that a reduction in membrane ergosterol composition or its replacement by other types of sterols, such as lanosterol, contributes to the reduced susceptibility to azoles and polyenes. Azole resistance of $C$. albicans has also been shown to be associated with increased expression of ERG11 (Lupetti et al., 2002; Morschhäuser, 2002). Some authors have also suggested that increased expression of ERG11 in the presence of an azole could be a feedback mechanism to compensate for the reduced ergosterol content. Our study showed that ERG11 expression in biofilms is significantly upregulated on challenge with ketoconazole but not with other antifungals. Therefore, we speculate that ERG11 might play a role in the azole resistance of $C$. albicans biofilms but not with other classes of drugs.

The glucan synthase gene FKS1, which encodes $1,3-\beta$-Dglucan, is associated with resistance to amphotericin $B$ and anidulafungin in Candida biofilms (Nett et al., 2010). We noted that FKS1 expression was significantly upregulated after treatment of C. albicans biofilms with amphotericin B and caspofungin. Therefore, it is conceivable that these biofilms rapidly increase glucan synthesis in response to noxious environmental stress exerted by antifungal agents. It is also known that point mutations or hot spots in the FKS1 gene may cause higher-order resistance, although some studies have presented conflicting evidence (Walker et al., 2010). This is a novel area that could be explored further to understand the mechanism of higher-order drug resistance in C. albicans biofilms.

PIL1 expression was significantly reduced on challenge with caspofungin and amphotericin B. It has been proposed that PIL1 is associated with $\beta$-1,3-glucan synthase and plays a role in its regulation (Edlind \& Katiyar, 2004). A protein complex containing both Pillp and Fkslp proteins has been identified (Gavin et al., 2002). Therefore, our results suggest that Pillp and Fkslp have opposing effects on the regulation of glucan synthesis, particularly in response to caspofungin and amphotericin B. At present, we are in the process of generating and characterizing PIL1 mutants. Understanding the roles of PIL1 and FKS1 may have implications in developing and modifying echinocandin-based regimens to eliminate C. albicans biofilms more efficiently.

Finally, MDR1 expression increased significantly when Candida biofilms were treated with amphotericin B. Because the amphotericin B molecule is too large for drug efflux pumps to expel it, increased MDR1 expression is unlikely to result in increased expulsion of this antifungal out of the cell. Amphotericin B kills fungi by acting on ergosterol in the fungal cell membrane to increase membrane permeability (Vanden Bossche et al., 1994). In addition to this primary action, amphotericin $B$ exerts oxidative stress on the fungal cells (Brajtburg et al., 1990; Ellis, 2002; Vanden Bossche et al., 1994). It is known that upregulation of the MDR1 gene is influenced by two regulatory elements: the hydrogen peroxide-responsive element and the benomylresponsive element (Rognon et al., 2006). Therefore, it is possible that increased expression of MDR1 is a consequence of oxidative damage induced by amphotericin B, perhaps as part of an oxidative defence system for protecting cells. This possible mechanism is supported by the fact that expression of MDR1 was not significantly altered by the other two drugs, caspofungin and ketoconazole, which do not cause oxidative damage to fungal cells. MDR1 overexpression has been linked to oxidative resistance in C. albicans (Kusch et al., 2004). We propose that an increase in MDR1 expression in Candida biofilms is a protective response to oxidative damage caused by amphotericin B but is not necessarily a resistance mechanism.

In conclusion, resistance of $C$. albicans biofilms to different classes of antifungals seems to be associated with transcriptional regulation of different drug-resistance genes. The data presented here shed some new light on drug resistance in C. albicans biofilms and lay a foundation for future large-scale genome-wide expression analysis.

\section{ACKNOWLEDGEMENTS}

This work was supported by the Hong Kong Research Grants Council, RGC no. HKU $7624 / 06 \mathrm{M}$ to L.P.S. and HKU internal funding 200907176121 to C.J.S. We thank Dr Trevor Lane for editorial assistance.

\section{REFERENCES}

Baillie, G. S. \& Douglas, L. J. (1999). Candida biofilms and their susceptibility to antifungal agents. Methods Enzymol 310, 644-656.

Beck-Sagué, C. M., Jarvis, W. R. \& National Nosocomial Infections Surveillance System (1993). Secular trends in the epidemiology of nosocomial fungal infections in the United States, 1980-1990. J Infect Dis 167, 1247-1251.

Beggs, W. H. (1989). Development of phenotypic resistance to direct lethal miconazole action by Candida albicans entering stationary phase. Mycopathologia 108, 201-206.

Brajtburg, J., Powderly, W. G., Kobayashi, G. S. \& Medoff, G. (1990). Amphotericin B: current understanding of mechanisms of action. Antimicrob Agents Chemother 34, 183-188.

Chandra, J., Mukherjee, P. K., Leidich, S. D., Faddoul, F. F., Hoyer, L. L., Douglas, L. J. \& Ghannoum, M. A. (2001). Antifungal resistance of candidal biofilms formed on denture acrylic in vitro. J Dent Res $\mathbf{8 0}$, 903-908.

Edlind, T. D. \& Katiyar, S. K. (2004). The echinocandin "target" identified by cross-linking is a homolog of Pill and Lsp1, sphingolipid-dependent regulators of cell wall integrity signaling. Antimicrob Agents Chemother 48, 4491. 
Ellepola, A. N. \& Samaranayake, L. P. (2001). Adjunctive use of chlorhexidine in oral candidoses: a review. Oral Dis 7, 11-17.

Ellis, D. (2002). Amphotericin B: spectrum and resistance. J Antimicrob Chemother 49 (Suppl. 1), 7-10.

Franz, R., Kelly, S. L., Lamb, D. C., Kelly, D. E., Ruhnke, M. \& Morschhäuser, J. (1998). Multiple molecular mechanisms contribute to a stepwise development of fluconazole resistance in clinical Candida albicans strains. Antimicrob Agents Chemother 42, 30653072.

Franz, R., Ruhnke, M. \& Morschhäuser, J. (1999). Molecular aspects of fluconazole resistance development in Candida albicans. Mycoses 42, 453-458.

Gavin, A.-C., Bösche, M., Krause, R., Grandi, P., Marzioch, M., Bauer, A., Schultz, J., Rick, J. M., Michon, A. M. \& other authors (2002). Functional organization of the yeast proteome by systematic analysis of protein complexes. Nature 415, 141-147.

Holmes, A. R., Lin, Y.-H., Niimi, K., Lamping, E., Keniya, M., Niimi, M., Tanabe, K., Monk, B. C. \& Cannon, R. D. (2008). ABC transporter Cdr1p contributes more than Cdr2p does to fluconazole efflux in fluconazole-resistant Candida albicans clinical isolates. Antimicrob Agents Chemother 52, 3851-3862.

Jayatilake, J. A., Samaranayake, L. P., Lu, Q. \& Jin, L. J. (2007). IL-1 $\alpha$, IL-1ra and IL- 8 are differentially induced by Candida in experimental oral candidiasis. Oral Dis 13, 426-433.

Jin, Y., Samaranayake, L. P., Samaranayake, Y. \& Yip, H. K. (2004). Biofilm formation of Candida albicans is variably affected by saliva and dietary sugars. Arch Oral Biol 49, 789-798.

Kusch, H., Biswas, K., Schwanfelder, S., Engelmann, S., Rogers, P. D., Hecker, M. \& Morschhäuser, J. (2004). A proteomic approach to understanding the development of multidrug-resistant Candida albicans strains. Mol Genet Genomics 271, 554-565.

Lopez-Ribot, J. L., McAtee, R. K., Lee, L. N., Kirkpatrick, W. R., White, T. C., Sanglard, D. \& Patterson, T. F. (1998). Distinct patterns of gene expression associated with development of fluconazole resistance in serial Candida albicans isolates from human immunodeficiency virusinfected patients with oropharyngeal candidiasis. Antimicrob Agents Chemother 42, 2932-2937.

Lu, Q., Jayatilake, J. A., Samaranayake, L. P. \& Jin, L. (2006). Hyphal invasion of Candida albicans inhibits the expression of human $\beta$ defensins in experimental oral candidiasis. J Invest Dermatol 126, 2049-2056.

Lupetti, A., Danesi, R., Campa, M., Del Tacca, M. \& Kelly, S. (2002). Molecular basis of resistance to azole antifungals. Trends Mol Med 8, 76-81.

Mateus, C., Crow, S. A., Jr \& Ahearn, D. G. (2004). Adherence of Candida albicans to silicone induces immediate enhanced tolerance to fluconazole. Antimicrob Agents Chemother 48, 3358-3366.

Morschhäuser, J. (2002). The genetic basis of fluconazole resistance development in Candida albicans. Biochim Biophys Acta 1587, 240-248.

Mukherjee, P. K., Chandra, J., Kuhn, D. M. \& Ghannoum, M. A. (2003). Mechanism of fluconazole resistance in Candida albicans biofilms: phase-specific role of efflux pumps and membrane sterols. Infect Immun 71, 4333-4340.

Nakamura, K., Niimi, M., Niimi, K., Holmes, A. R., Yates, J. E., Decottignies, A., Monk, B. C., Goffeau, A. \& Cannon, R. D. (2001). Functional expression of Candida albicans drug efflux pump Cdrlp in a Saccharomyces cerevisiae strain deficient in membrane transporters. Antimicrob Agents Chemother 45, 3366-3374.

Navarro-Garcia, F., Sánchez, M., Nombela, C. \& Pla, J. (2001). Virulence genes in the pathogenic yeast Candida albicans. FEMS Microbiol Rev 25, 245-268.
Nett, J. E., Crawford, K., Marchillo, K. \& Andes, D. R. (2010). Role of Fkslp and matrix glucan in Candida albicans biofilm resistance to an echinocandin, pyrimidine, and polyene. Antimicrob Agents Chemother 54, 3505-3508.

Perea, S., López-Ribot, J. L., Kirkpatrick, W. R., McAtee, R. K., Santillán, R. A., Martínez, M., Calabrese, D., Sanglard, D. \& Patterson, T. F. (2001). Prevalence of molecular mechanisms of resistance to azole antifungal agents in Candida albicans strains displaying high-level fluconazole resistance isolated from human immunodeficiency virusinfected patients. Antimicrob Agents Chemother 45, 2676-2684.

Pfaffl, M. W. (2001). A new mathematical model for relative quantification in real-time RT-PCR. Nucleic Acids Res 29, e45.

Pfaller, M. A. \& Diekema, D. J. (2007). Epidemiology of invasive candidiasis: a persistent public health problem. Clin Microbiol Rev 20, 133-163.

Prasad, R. \& Kapoor, K. (2004). Multidrug resistance in yeast Candida. Int Rev Cytol 242, 215-248.

Ramage, G., Bachmann, S., Patterson, T. F., Wickes, B. L. \& LópezRibot, J. L. (2002). Investigation of multidrug efflux pumps in relation to fluconazole resistance in Candida albicans biofilms. J Antimicrob Chemother 49, 973-980.

Rognon, B., Kozovska, Z., Coste, A. T., Pardini, G. \& Sanglard, D. (2006). Identification of promoter elements responsible for the regulation of MDR1 from Candida albicans, a major facilitator transporter involved in azole resistance. Microbiology 152, 37013722.

Ruhnke, M., Hartwig, K. \& Kofla, G. (2008). New options for treatment of candidaemia in critically ill patients. Clin Microbiol Infect 14 (Suppl. 4), 46-54.

Samaranayake, L. P. \& MacFarlane, T. W. (1990). Oral Candidosis. London: Wright.

Samaranayake, Y. H., Dassanayake, R. S., Jayatilake, J. A., Cheung, B. P., Yau, J. Y., Yeung, K. W. \& Samaranayake, L. P. (2005). Phospholipase B enzyme expression is not associated with other virulence attributes in Candida albicans isolates from patients with human immunodeficiency virus infection. J Med Microbiol 54, 583593.

Sanglard, D., Kuchler, K., Ischer, F., Pagani, J. L., Monod, M. \& Bille, J. (1995). Mechanisms of resistance to azole antifungal agents in Candida albicans isolates from AIDS patients involve specific multidrug transporters. Antimicrob Agents Chemother 39, 23782386.

Seneviratne, C. J., Jin, L. \& Samaranayake, L. P. (2008a). Biofilm lifestyle of Candida: a mini review. Oral Dis 14, 582-590.

Seneviratne, C. J., Jin, L. J., Samaranayake, Y. H. \& Samaranayake, L. P. (2008b). Cell density and cell aging as factors modulating antifungal resistance of Candida albicans biofilms. Antimicrob Agents Chemother 52, 3259-3266.

Seneviratne, C. J., Wang, Y., Jin, L., Abiko, Y. \& Samaranayake, L. P. (2008c). Candida albicans biofilm formation is associated with increased anti-oxidative capacities. Proteomics 8, 2936-2947.

Tumbarello, M., Posteraro, B., Trecarichi, E. M., Fiori, B., Rossi, M., Porta, R., de Gaetano Donati, K., La Sorda, M., Spanu, T. \& other authors (2007). Biofilm production by Candida species and inadequate antifungal therapy as predictors of mortality for patients with candidemia. J Clin Microbiol 45, 1843-1850.

Vanden Bossche, H., Marichal, P. \& Odds, F. C. (1994). Molecular mechanisms of drug resistance in fungi. Trends Microbiol 2, 393400.

Walker, L. A., Gow, N. A. \& Munro, C. A. (2010). Fungal echinocandin resistance. Fungal Genet Biol 47, 117-126. 
White, T. C. (1997). Increased mRNA levels of ERG16, CDR, and $M D R 1$ correlate with increases in azole resistance in Candida albicans isolates from a patient infected with human immunodeficiency virus. Antimicrob Agents Chemother 41, 1482-1487.
Wisplinghoff, H., Bischoff, T., Tallent, S. M., Seifert, H., Wenzel, R. P. \& Edmond, M. B. (2004). Nosocomial bloodstream infections in US hospitals: analysis of 24,179 cases from a prospective nationwide surveillance study. Clin Infect Dis 39, 309-317. 